\title{
First principles investigation of defect energy levels at semiconductor- oxide interfaces: Oxygen vacancies and hydrogen interstitials in the $\mathrm{Si}-\mathrm{SiO}_{2}-\mathrm{HfO}_{2}$ stack
}

\author{
Peter Broqvist, ${ }^{\text {a) }}$ Audrius Alkauskas, Julien Godet, ${ }^{\text {b) }}$ and Alfredo Pasquarello \\ Institute of Theoretical Physics, Ecole Polytechnique Fédérale de Lausanne (EPFL), CH-1015 Lausanne, \\ Switzerland and Institut Romand de Recherche Numérique en Physique des Matériaux (IRRMA), CH- \\ 1015 Lausanne, Switzerland
}

(Received 8 August 2008; accepted 24 September 2008; published online 16 March 2009)

\begin{abstract}
We introduce a scheme for the calculation of band offsets and defect energy levels at semiconductor-oxide interfaces. Our scheme is based on the use of realistic atomistic models of the interface structure and of hybrid functionals for the evaluation of the electronic structure. This scheme is herein applied to the technologically relevant $\mathrm{Si}-\mathrm{SiO}_{2}-\mathrm{HfO}_{2}$ stack. Calculated band offsets show a very good agreement with experimental values. In particular, we focus on the energy levels of the oxygen vacancy defect and the interstitial hydrogen impurity. The defect levels are aligned with respect to the interface band structure and determined for varying location in the dielectric stack. The most stable charge states are identified as the Fermi level sweeps through the silicon band gap. (C) 2009 American Institute of Physics. [DOI: 10.1063/1.3055347]
\end{abstract}

\section{INTRODUCTION}

The replacement of $\mathrm{SiO}_{2}$ with an oxide of higher dielectric constant (high- $\kappa$ ) (Ref. 1) has dramatically brought to the forefront our need for developing a more extended understanding of the atomic and electronic properties of typical defects in the new oxides. First principles modeling approaches are expected to provide a description of the atomic configurations of the unknown defects together with a characterization of their electronic energy levels with respect to the relevant band edges. However, the achievement of this target still faces several difficulties. ${ }^{2}$

Two aspects need particular attention. The first concerns the modeling of the interfacial transition region, which is generally characterized by a nontrivial connection between the crystalline semiconductor and the disordered oxide. To achieve a reliable band alignment at the interface, it is critical to provide a realistic description of the atomistic structure in this region. ${ }^{3-5}$ Indeed, previous density functional studies on crystalline-crystalline interfaces have revealed a strong sensitivity of the band offsets on the adopted model of the interfacial bonding pattern. ${ }^{3,4}$ Therefore, it is important to ensure that the transition region is smooth in terms of bond parameters and coordination. ${ }^{5-9}$ For the $\mathrm{Si}-\mathrm{SiO}_{2}$ interface, such a choice led to variations of $0.1-0.2 \mathrm{eV}$ in the calculated band offsets. ${ }^{10,11}$ Furthermore, it is important to describe the amorphous nature of the oxide in order to capture the specific properties of interfacial defects. ${ }^{5,12-14}$

The second aspect concerns the method for describing the electronic structure. Density functional calculations of band offsets describe the electronic and atomic arrangements at the interface in a self-consistent way. ${ }^{15-19}$ However, the most common approximations to the exchange-correlation

\footnotetext{
${ }^{a)}$ Electronic mail: peter.broqvist @epfl.ch.

${ }^{b}$ Present address: PhyMat, University of Poitiers - CMRS, SP2MI - BP 30179, 86962 Futuroscope, France.
}

energy, i.e., the generalized-gradient approximation and the local density approximation, lead to significant underestimations of band gaps, thereby impairing the reliability of calculated band offsets and defect levels. Previous theoretical work shows that band-offset errors for semiconductor-oxide interfaces can reach several $\mathrm{eV}^{3,8,9,20}$ Moreover, this discrepancy directly affects the determination of defect levels, making comparisons with experiment ambiguous. Therefore, it is ineluctable to address the band-gap problem going beyond semilocal approximations to density functional theory. Many-body perturbation theory in the $G W$ approximation is the method of choice for calculating band offsets ${ }^{21,22}$ and defect levels ${ }^{23,24}$ but remains computationally demanding and therefore limited to small-sized systems. For instance, the study of realistic semiconductor-oxide interfaces in which the oxide is noncrystalline ${ }^{5,8,9}$ is severely hindered.

In this work, we address band offsets and defect levels at semiconductor-oxide interfaces through the use of reliable interface models and hybrid functionals. These functionals incorporate a fraction of Hartree-Fock exchange, leading to a higher accuracy ${ }^{25}$ and improved band gaps ${ }^{26,27}$ compared to semilocal functionals. We focus on the technologically relevant $\mathrm{Si}-\mathrm{SiO}_{2}-\mathrm{HfO}_{2}$ stack. First, we model the interface structure accounting for the occurrence of a $\mathrm{SiO}_{2}$ interlayer and for the amorphous nature of the near-interface oxide and determine the band alignment at the interface. Then, we consider two kinds of oxide defects, the oxygen vacancy and the interstitial hydrogen, and align their energy levels to the interface band structure. For these defects, we calculate the formation energies for varying location across the dielectric stack and determine the stable charge states as the Fermi level sweeps through the silicon band gap.

\section{METHODS}

In the present work, the structural models were generated and structurally relaxed through the use of the semilocal 


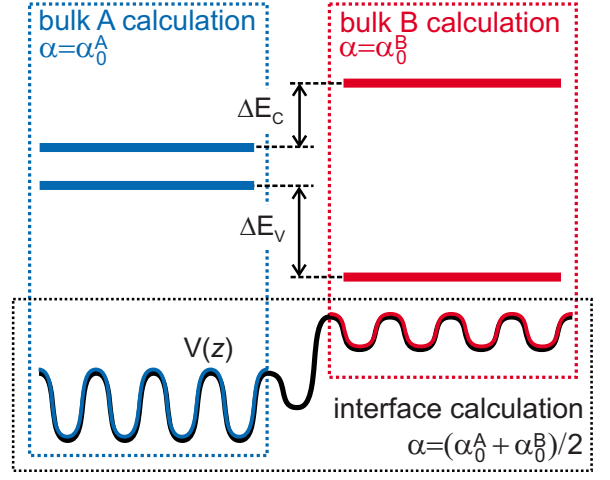

FIG. 1. (Color online) Schematic presenting the adopted band alignment scheme. The scheme is based on the choice of $\alpha$ reproducing the experimental band gap of each interface component (Refs. 5 and 31).

approximation for the exchange-correlation energy proposed by Perdew, Burke, and Ernzerhof (PBE) ${ }^{28}$ For the electronic structure, we used the class of hybrid density functionals which are based on the semilocal PBE approximation and are obtained by replacing a fraction $\alpha$ of PBE exchange with Hartree-Fock exchange. ${ }^{29}$ For each material, we used the fraction $\alpha$ that reproduced the experimental band gap.

We calculated the band offsets by aligning the band extrema of the bulk interface components through a local reference level at the interface. ${ }^{30}$ This procedure relies on the weak dependence of the offset of the local electrostatic potential across the interface on $\alpha .{ }^{5,31}$ Hence, the band structures of the individual bulk components are obtained through the use of optimal $\alpha$ 's, while the choice of $\alpha$ in the interface calculation is not critical. The band alignment procedure is schematically illustrated in Fig. 1 and has been found to yield band offsets in excellent agreement with experimental values for a series of interface systems. ${ }^{5,31}$

As far as the defect levels are concerned, a recent investigation has shown that they are positioned on an energy scale that is practically unaffected by band-gap renormalization. ${ }^{32}$ Hence, hybrid functionals with optimized $\alpha$ modify the position of the band edges but do not significantly affect the relative location of the defect levels in wide band-gap oxides. The application of this scheme to wellcharacterized defects yields excellent agreement with the experiment. $^{32,33}$

The results presented here were obtained with a planewave pseudopotential approach. For the computational settings, we refer to Refs. 5, 20, and 34. We used the QUANTUMESPRESSO (Ref. 35) and CPMD (Ref. 36) packages.

\section{STRUCTURAL MODELS}

To describe the structural environment in the $\mathrm{Si}-\mathrm{SiO}_{2}-\mathrm{HfO}_{2}$ stack, we considered both the bulk and the interface models (Fig. 2). To represent the various stack components, we used bulk models of amorphous $\mathrm{SiO}_{2}$ (Refs. 37 and 38) and amorphous $\left(\mathrm{HfO}_{2}\right)_{x}\left(\mathrm{SiO}_{2}\right)_{1-x} \cdot{ }^{34,39,40}$ The bulk oxide was modeled by monoclinic $\mathrm{HfO}_{2}\left(m-\mathrm{HfO}_{2}\right) .{ }^{41} \mathrm{We}$ generated a model interface between crystalline silicon and amorphous $\mathrm{HfO}_{2}$ including the $\mathrm{SiO}_{2}$ interlayer through $a b$ (a)

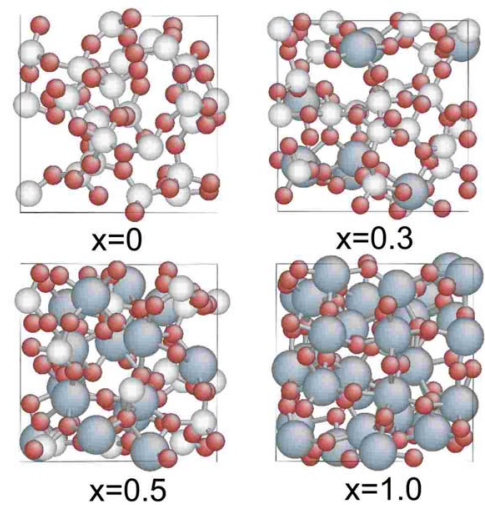

(b)

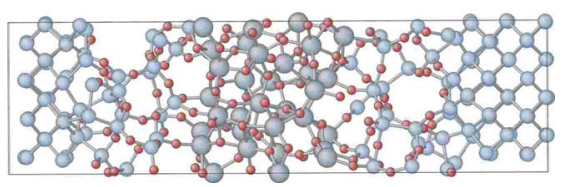

FIG. 2. (Color online) (a) Bulk models of amorphous $\left(\mathrm{HfO}_{2}\right)_{x}\left(\mathrm{SiO}_{2}\right)_{1-x}$ (Refs. 34 and 37-40) and (b) interface model of the $\mathrm{Si}-\mathrm{SiO}_{2}-\mathrm{HfO}_{2}$ stack (Ref. 5).

initio molecular dynamics. ${ }^{5}$ The structural properties of these models will be discussed in the following.

\section{A. Bulk models}

We generated models for $\left(\mathrm{HfO}_{2}\right)_{x}\left(\mathrm{SiO}_{2}\right)_{1-x}$ with $x=0$, $0.3,0.5$, and $1 . .^{34,37-40}$ The models were generated through constant volume simulations carried out with densities corresponding to zirconium silicates. We used experimental data up to $x=0.5$ and an extrapolation of the linear experimental trend for amorphous $\mathrm{HfO}_{2}{ }^{42}$ Hence, the adopted densities were $2.2,4.2,5.8$, and $9.1 \mathrm{~g} / \mathrm{cm}^{3}$ for $x=0,0.3,0.5$, and 1 , respectively. The initial atomic configurations of our simulations were obtained from preliminary classical molecular dynamics runs. The final structures are the result of thermal quenches from the melt at the $a b$ initio level of theory.

The generated structures are displayed in Fig. 2(a). In our models, only $\mathrm{Hf}-\mathrm{O}$ and $\mathrm{Si}-\mathrm{O}$ bonds occurred. We have characterized the structural evolution from $\mathrm{SiO}_{2}$ to $\mathrm{HfO}_{2}$ through the average atomic coordination numbers calculated by integrating the pair correlation functions until their first minima. In amorphous $\mathrm{SiO}_{2}$, silicon and oxygen were found to be fourfold and twofold coordinated, respectively. Silicon remains fourfold coordinated in all our silicate models $(x$ $=0.3,0.5)$. The average $\mathrm{Si}-\mathrm{O}$ bond lengths increase from $1.64 \AA$ at $x=0$ to $1.66 \AA$ at $x=0.5$, showing a weak dependence on Hf content. For the hafnium coordination, we obtained 5.1, 5.9, and 6.2 for $x=0.3,0.5$, and 1, respectively. The increase in coordination is accompanied by a small elongation of the average $\mathrm{Hf}-\mathrm{O}$ bond length: $2.10 \AA$ at $x=0.3$, $2.13 \AA$ at $x=0.5$, and $2.14 \AA$ at $x=1$. The average coordination of the oxygen atoms increases steadily with Hf content, giving coordination numbers of 2.0, 2.2, 2.5, and 3.0 for $x$ $=0,0.3,0.5$, and 1 , respectively. We note that with increasing Hf content, the hafnium and oxygen coordinations approach those of monoclinic $\mathrm{HfO}_{2}$, which corresponds to the most 
stable crystalline phase. However, the hafnium and oxygen coordinations in our model of amorphous $\mathrm{HfO}_{2}$ still remain below the average crystalline values of 7 and 3.5, respectively.

\section{B. Interface model}

At the $\mathrm{Si}-\mathrm{HfO}_{2}$ interface, the hafnium oxide is amorphous when deposited at low temperatures but transforms to a polycrystalline form upon the thermal annealing required for dopant activation. ${ }^{43}$ The transition is characterized by the formation of an amorphous interlayer composed of $\mathrm{SiO}_{2}{ }^{43}$ These experimental aspects have guided the generation of an atomistic interface model.

We generated a model interface between crystalline silicon and amorphous $\mathrm{HfO}_{2}$ including the amorphous $\mathrm{SiO}_{2}$ interlayer through ab initio molecular dynamics. ${ }^{5}$ To avoid the description of outer surfaces, we adopted a superlattice geometry. The attachment of the $\mathrm{SiO}_{2}$ to the $\mathrm{Si}(100)$ substrate was based on previous models of the $\mathrm{Si}-\mathrm{SiO}_{2}$ interface, which account for photoemission and ion-scattering experiments. ${ }^{6,7}$ We kept $7 \AA$ of $\mathrm{SiO}_{2}$ on either side of a 10 ML thick $\mathrm{Si}(100)$ substrate. The region between the $\mathrm{SiO}_{2}$ terminations was filled with a $\mathrm{HfO}_{2}$ layer of $\sim 1.2 \mathrm{~nm}$ in accordance with electron counting rules ${ }^{3}$ and the experimental density ${ }^{44}$ of $\sim 9 \mathrm{~g} / \mathrm{cm}^{3}$. The model consisted of 282 atoms. The final structure was generated by evolving the $\mathrm{HfO}_{2}$ structure at a temperature of $3000 \mathrm{~K}$, while initially keeping the boundary $\mathrm{SiO}_{2}$ layers frozen. At this temperature, diffusive motion was observed in the $\mathrm{HfO}_{2}$ layer on the time scale of our simulation ( $8 \mathrm{ps})$. After the high-temperature treatment, we applied a thermal quench to $T=0 \mathrm{~K}$ which allowed the full structural model to relax.

The final model structure is shown in Fig. 2(b). The mass density across the $\mathrm{HfO}_{2}$ layer remained close to the targeted value of $9 \mathrm{~g} / \mathrm{cm}^{3}$ and the electronic density of states revealed no localized defect states in the band gap, thereby ensuring a defect free amorphous $\mathrm{HfO}_{2}$ thin film. The average atomic oxygen concentration is fairly constant at around $2 / 3$ throughout the oxide stack, while the silicon and hafnium concentrations interchange at the $\mathrm{SiO}_{2}-\mathrm{HfO}_{2}$ interface. As this interface is crossed the oxygen coordination increases from 2 in the $\mathrm{SiO}_{2}$ to 3 in the $\mathrm{HfO}_{2}$. At the $\mathrm{SiO}_{2}-\mathrm{HfO}_{2}$ interface, the oxygen atoms primarily occur in twofold-coordinated local arrangements with hafnium and silicon atoms in their first-neighbor shell. However, other arrangements are also found such as threefold coordinated oxygen atoms either with 2 hafnium and 1 silicon or with 1 hafnium and 2 silicon. The average $\mathrm{Hf}-\mathrm{O}$ bond length in the model interface is $2.1 \AA$ in good agreement with typical values for amorphous $\mathrm{HfO}_{2}$. The average hafnium coordination by oxygen increases from 5.7 in the vicinity of the interface to 6.2 in the middle of the $\mathrm{HfO}_{2}$ layer. The silicon atoms are always fourfold coordinated in our model, but their coordination by oxygen gradually increases across the $\mathrm{Si}-\mathrm{SiO}_{2}$ interface.

\section{BAND ALIGNMENTS}

Before calculating band offsets, we determined the optimal $\alpha$ that reproduced the band gap of each interface component. For silicon, we used $\alpha=0.11$ for which we obtained the well-characterized band gap of $1.17 \mathrm{eV}$. In the case of amorphous $\mathrm{SiO}_{2}$, an $\alpha$ of 0.34 was required to recover the measured band gap of $8.9 \mathrm{eV}$. While the experimental band gap of $m-\mathrm{HfO}_{2}$ is well characterized, the measured band gaps of the hafnium containing oxides $\left[\left(\mathrm{HfO}_{2}\right)_{x}\left(\mathrm{SiO}_{2}\right)_{1-x}\right]$ show a significant scatter. ${ }^{34}$ Density functional calculations reveal that the conduction band minimum in the latter materials is determined by hafnium states while the top of the valence band results from oxygen $2 p$ states. $^{34}$ Therefore, it is reasonable to take a constant value of $\alpha$ for the hafnium containing oxides used in this study $(x>30 \%)$ rather than to apply a linear extrapolation scheme between the optimal $\alpha$ for $\mathrm{SiO}_{2}$ and $m-\mathrm{HfO}_{2}$. For $m-\mathrm{HfO}_{2}$, the experimental band gap of 5.8 $\mathrm{eV}$ (Ref. 45) was recovered with $\alpha=0.15$. Using this $\alpha$ for crystalline $\mathrm{HfSiO}_{4}$ (hafnon) yielded a band gap of $7.0 \mathrm{eV}$ in reasonably good agreement with the experimental estimate of 6.5-7.0 eV. ${ }^{46}$ For amorphous $\mathrm{HfO}_{2}$ and $\mathrm{HfSiO}_{4}$, we obtained 5.4 and $5.6 \mathrm{eV}$, in similar good agreement with the experimental data. ${ }^{47,48}$

For the calculation of band offsets, we used local reference levels determined in the interface model to align the band extrema of the various stack components. ${ }^{30}$ The procedure is schematically illustrated in Fig. 1. For silicon, the band structure was aligned through the electrostatic potential, while the deep oxygen $2 s$ and hafnium $5 s$ levels were used for the $\mathrm{SiO}_{2}$ and hafnium containing components, respectively. The resulting band alignment is schematically illustrated in Fig. 3. In particular, we obtained a $4.4 \mathrm{eV}$ valence band offset between silicon and amorphous $\mathrm{SiO}_{2}$ in accord with the measured value. ${ }^{49,50}$ For the interface between silicon and monoclinic $\mathrm{HfO}_{2}$, we obtained a valence band offset of $2.9 \mathrm{eV}$, very close to the measured value of 3.0 $\mathrm{eV}^{51,52}$ This level of agreement provides confidence for the quantitative determination of defect levels with respect to the silicon band edges.

\section{OXIDE DEFECTS}

The generated models were used to study two common oxide defects: the oxygen vacancy and the hydrogen interstitial. The oxygen vacancy has been the object of extensive studies in literature ${ }^{41,53,54}$ because it is suspected to be at the origin of the Fermi-level pinning in $p$-type polysilicon gate stacks. ${ }^{55,56}$ The hydrogen interstitial is ubiquitous at such interfaces. While it is known to play a beneficial role in passivating dangling bonds at the $\mathrm{Si}-\mathrm{SiO}_{2}$ interface, this impurity may be at the origin of the negative bias temperature instability $^{57}$ and the positive charging of the gate oxide. ${ }^{57,58}$ To develop a deeper understanding of the role of these defects, we address here their stable charge states and their locations across the dielectric stack.

\section{A. Oxygen vacancy}

We examined the relationship between the oxygen vacancy and the Fermi-level pinning. ${ }^{5}$ The oxygen vacancy oc- 


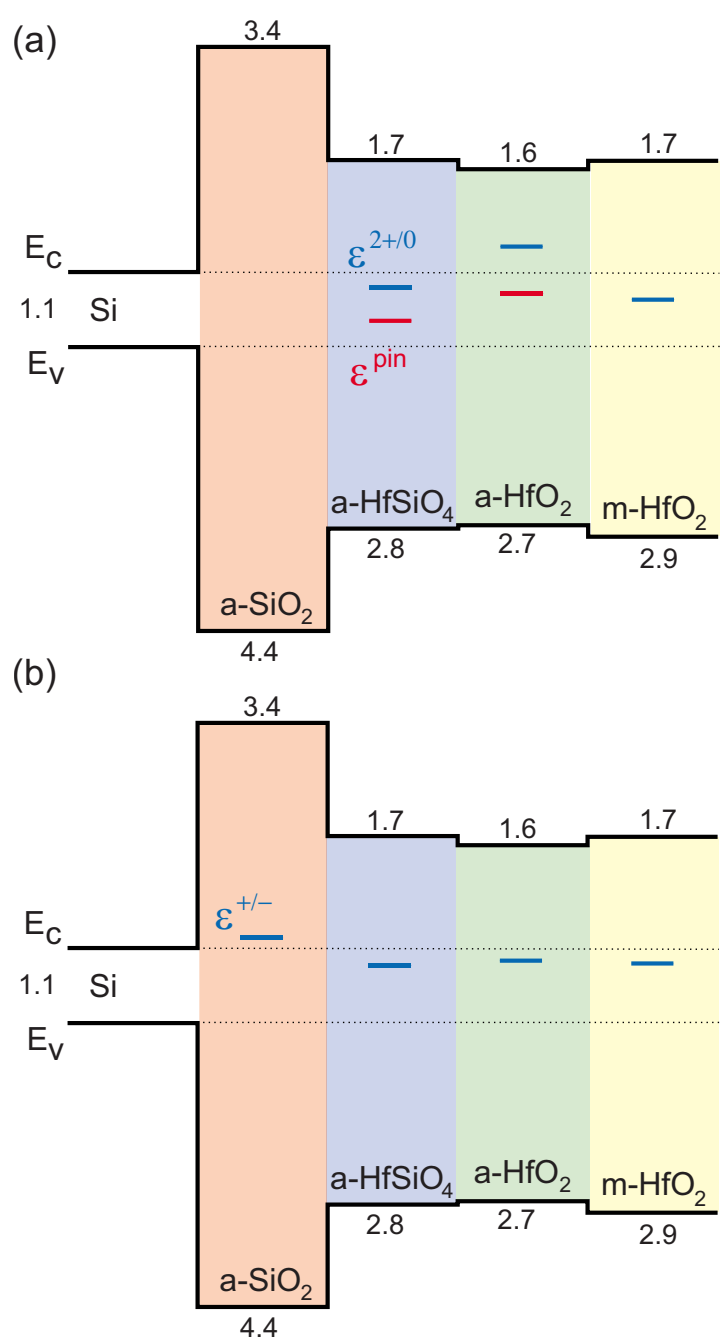

FIG. 3. (Color online) Band alignments in the $\mathrm{Si}-\mathrm{SiO}_{2}-\mathrm{HfO}_{2}$ stack composed of $\mathrm{Si}$, amorphous $\mathrm{SiO}_{2}$, amorphous $\mathrm{HfSiO}_{4}$, amorphous $\mathrm{HfO}_{2}$, and monoclinic $\mathrm{HfO}_{2}$. Defect levels associated to (a) the oxygen vacancy and (b) the hydrogen interstitial are indicated. The pinning levels $\left(\epsilon_{\text {pin }}\right)$ associated with the oxygen vacancies are also shown.

curs in several charge states, but the neutral and the doubly positive charge states are the most relevant for Fermi levels falling close to the silicon band edges. Formation energies of these charge states were calculated in each of the hafnium containing components of the gate stack. For both charge states, the calculated formation energy is lower in the amorphous components than in monoclinic $\mathrm{HfO}_{2}$. The charge transition levels $\epsilon^{2+/ 0}$ for each component are given in the band diagram of Fig. 3(a). For $p$-type gates, the most stable oxygen vacancy is found for the doubly positive charge state in the amorphous $\mathrm{HfO}_{2}$ layer. In consideration of the low activation energies for vacancy diffusion across monoclinic $\mathrm{HfO}_{2}(0.7 \mathrm{eV}),{ }^{59}$ this result indicates that oxygen vacancies accumulate in this layer, consistent with the experimental observations locating charged defects in the vicinity of the polysilicon gate. ${ }^{60}$

According to the current understanding of Fermi-level pinning in these gate stacks, the oxygen vacancy formation is accompanied by a silicon oxidation reaction. ${ }^{55,56}$ The vacancy generation proceeds as long as this reaction is exothermic $(\Delta G<0),{ }^{55,56}$ thereby fixing the pinning level through the condition $\Delta G=0$. Our results indicate that oxygen vacancies in monoclinic $\mathrm{HfO}_{2}$ do not lead to Fermi-level pinning. At variance, the amorphous components show pinning levels within the silicon band gap, which account for the position of measured levels ${ }^{55,56}$ Hence, our results for both the energetics and the Fermi-level pinning indicate that oxygen vacancies locate in the noncrystalline transition regions of the gate stack.

\section{B. Hydrogen interstitial}

We studied the energetics of various structural configurations and charge states of hydrogen in the $\mathrm{Si}-\mathrm{SiO}_{2}-\mathrm{HfO}_{2}$ stack. ${ }^{20}$ Hydrogen is found to be amphoteric with a $\epsilon^{+/-}$ charge transition level lying close to the $\mathrm{Si}$ conduction band minimum for all stack components [cf. Fig. 3(b)]. In $\mathrm{SiO}_{2}$, the charge transition level is located just above the silicon conduction band minimum, whereas it is located in the upper part of the silicon band gap for the hafnium-containing stack components. In the positive charge state, the hydrogen interstitial is generally most stable when attached to an oxygen atom which forms two bonds with the oxide network. The negatively charged state corresponds to the hydrogen attached to either silicon or a hafnium atom, the latter being energetically more favorable.

Our results indicate that the proton is the stable charge state of hydrogen across the $\mathrm{Si}-\mathrm{SiO}_{2}-\mathrm{HfO}_{2}$ stack for most Fermi levels in the Si band gap in accord with the previous results obtained at the PBE level of theory. ${ }^{20}$ The most stable configurations are found in two distinct regions: at the $\mathrm{Si}-\mathrm{SiO}_{2}$ interface and toward the $\mathrm{HfO}_{2}$ layer. At the $\mathrm{Si}-\mathrm{SiO}_{2}$ interface, the proton induces the formation of deep positive charge traps consisting of threefold coordinated oxygen atoms. ${ }^{12}$ In the hafnium silicates, the most stable states consist of oxygen atoms that are twofold coordinated by hafnium. For instance, the energy of $\mathrm{Hf}-\mathrm{OH}^{+}-\mathrm{Hf}$ configurations is lower by as much as $1.3 \mathrm{eV}$ with respect to the energy of mobile protons in $\mathrm{SiO}_{2}{ }^{20}$ Furthermore, it is noteworthy that higher proton energies are obtained for oxygen atoms with higher coordinations, which predominate in bulk $\mathrm{HfO}_{2}$. Hence, we infer that protons in $\mathrm{Si}-\mathrm{SiO}_{2}-\mathrm{HfO}_{2}$ stacks are trapped either at the $\mathrm{Si}-\mathrm{SiO}_{2}$ interface or at the edge of the $\mathrm{HfO}_{2}$ layer. These results are consistent with the experiment. ${ }^{58}$

\section{CONCLUSION}

It is anticipated that as new materials solutions are screened in the microelectronic technology, the need for a theoretical tool for defect identification will prove ineluctable. In this work, we demonstrated a comprehensive theoretical scheme for determining the band alignment and defect energy levels at semiconductor-oxide interfaces. The scheme is based on the use of hybrid density functionals for the electronic structure and of realistic atomistic models of the interface and its bulk components.

We here applied the scheme to two oxide defects in the technologically important $\mathrm{Si}-\mathrm{SiO}_{2}-\mathrm{HfO}_{2}$ stack: the oxygen vacancy and the hydrogen interstitial. It is shown that the scheme reliably reproduces the experimental band alignment 
and that it allows one to locate the defect energy levels with respect to the semiconductor and oxide band edges. The stable charge states of the defects and their location within the dielectric stack can thus be studied as the Fermi level sweeps through the silicon band gap. In particular, our scheme identifies the oxygen vacancy located in the amorphous transition region as a potential candidate for causing Fermi-level pinning in $p$-type gates.

\section{ACKNOWLEDGMENTS}

Financial support from the Swiss National Science Foundation (Grant Nos. 200020-119733 and 200020111747) is acknowledged. Most of the calculations were performed on the BlueGene of EPFL. We also used the facilities at DIT-EPFL, CSEA-EPFL, and CSCS.

${ }^{1}$ J. Robertson, Eur. Phys. J.: Appl. Phys. 28, 265 (2004).

${ }^{2}$ A. Pasquarello and A. M. Stoneham, J. Phys.: Condens. Matter 17, V1 (2005).

${ }^{3}$ P. W. Peacock and J. Robertson, Phys. Rev. Lett. 92, 057601 (2004).

${ }^{4}$ O. Sharia, A. A. Demkov, G. Bersuker, and B. H. Lee, Phys. Rev. B 75, 035306 (2007)

${ }^{5}$ P. Broqvist, A. Alkauskas, and A. Pasquarello, Appl. Phys. Lett. 92, 132911 (2008).

${ }^{6}$ A. Bongiorno, A. Pasquarello, M. S. Hybertsen, and L. C. Feldman, Phys Rev. Lett. 90, 186101 (2003).

${ }^{7}$ A. Bongiorno and A. Pasquarello, Appl. Phys. Lett. 83, 1417 (2003)

${ }^{8}$ F. Giustino and A. Pasquarello, Phys. Rev. Lett. 95, 187402 (2005).

${ }^{9}$ F. Devynck, F. Giustino, P. Broqvist, and A. Pasquarello, Phys. Rev. B 76, 075351 (2007)

${ }^{10}$ F. Giustino, A. Bongiorno, and A. Pasquarello, Appl. Phys. Lett. 86, 192901 (2005)

${ }^{11}$ F. Giustino and A. Pasquarello, Surf. Sci. 586, 183 (2005).

${ }^{12}$ J. Godet, F. Giustino, and A. Pasquarello, Phys. Rev. Lett. 99, 126102 (2007).

${ }^{13}$ A. Bongiorno and A. Pasquarello, Phys. Rev. Lett. 88, 125901 (2002).

${ }^{14}$ A. Bongiorno and A. Pasquarello, Phys. Rev. B 70, 195312 (2004).

${ }^{15}$ G. A. Baraff, J. A. Appelbaum, and D. R. Hamann, Phys. Rev. Lett. 38, 237 (1977).

${ }^{16}$ W. E. Pickett, S. G. Louie, and M. L. Cohen, Phys. Rev. B 17, 815 (1978)

${ }^{17}$ C. G. Van de Walle and R. L. Martin, Phys. Rev. B 35, 8154 (1987).

${ }^{18}$ S.-H. Wei and A. Zunger, Appl. Phys. Lett. 72, 2011 (1998).

${ }^{19}$ N. Peressi, N. Binggeli, and A. Baldereschi, J. Phys. D 31, 1273 (1998).

${ }^{20}$ J. Godet, P. Broqvist, and A. Pasquarello, Appl. Phys. Lett. 91, 262901 (2007).

${ }^{21}$ S. B. Zhang, D. Tománek, S. G. Louie, M. L. Cohen, and M. S. Hybertsen, Solid State Commun. 66, 585 (1988).

${ }^{22}$ R. Shaltaf, G.-M. Rignanese, X. Gonze, F. Giustino, and A. Pasquarello, Phys. Rev. Lett. 100, 186401 (2008).

${ }^{23}$ S. Ismail-Beigi and S. G. Louie, Phys. Rev. Lett. 95, 156401 (2005).

${ }^{24}$ M. Hedström, A. Schindlmayr, G. Schwarz, and M. Scheffler, Phys. Rev. Lett. 97, 226401 (2006).

${ }^{25}$ L. A. Curtiss, P. C. Redfern, K. Raghavachari, and J. A. Pople, J. Chem. Phys. 109, 42 (1998).

${ }^{26}$ J. Muscat, A. Wander, and N. M. Harrison, Chem. Phys. Lett. 342, 397 (2001).

${ }^{27}$ J. Paier, M. Marsman, K. Hummer, G. Kresse, I. C. Gerber, and J. G.
Ángyán, J. Chem. Phys. 124, 154709 (2006); 125, 249901 (2006).

${ }^{28}$ J. P. Perdew, K. Burke, and M. Ernzerhof, Phys. Rev. Lett. 77, 3865 (1996).

${ }^{29}$ J. P. Perdew, M. Ernzerhof, and K. Burke, J. Chem. Phys. 105, 9982 (1996).

${ }^{30}$ C. G. Van de Walle and R. M. Martin, Phys. Rev. B 34, 5621 (1986).

${ }^{31}$ A. Alkauskas, P. Broqvist, F. Devynck, and A. Pasquarello, Phys. Rev. Lett. 101, 106802 (2008).

${ }^{32}$ A. Alkauskas, P. Broqvist, and A. Pasquarello, Phys. Rev. Lett. 101, 046405 (2008).

${ }^{33}$ P. Broqvist, A. Alkauskas, and A. Pasquarello, Phys. Rev. B 78, 075203 (2008).

${ }^{34}$ P. Broqvist and A. Pasquarello, Appl. Phys. Lett. 90, 082907 (2007).

${ }^{35}$ A. Pasquarello, K. Laasonen, R. Car, C. Lee, and D. Vanderbilt, Phys. Rev. Lett. 69, 1982 (1992); K. Laasonen, A. Pasquarello, R. Car, C. Lee, and D. Vanderbilt, Phys. Rev. B 47, 10142 (1993); we used the implementation provided by http://www.quantum-espresso.org/.

${ }^{36}$ CPMD, Copyright IBM Corp. 1990-2006, Copyright MPI für Festkörperforschung Stuttgart, 1997.

${ }^{37}$ J. Sarnthein, A. Pasquarello, and R. Car, Phys. Rev. Lett. 74, 4682 (1995).

${ }^{38}$ J. Sarnthein, A. Pasquarello, and R. Car, Phys. Rev. B 52, 12690 (1995).

${ }^{39}$ P. Broqvist and A. Pasquarello, Microelectron. Eng. 84, 2416 (2007).

${ }^{40}$ P. Broqvist and A. Pasquarello, Microelectron. Eng. 84, 2022 (2007).

${ }^{41}$ P. Broqvist and A. Pasquarello, Appl. Phys. Lett. 89, 262904 (2006).

${ }^{42}$ M. Nogami, J. Non-Cryst. Solids 69, 415 (1985).

${ }^{43}$ G. D. Wilk and D. A. Muller, Appl. Phys. Lett. 83, 3984 (2003).

${ }^{44}$ M. Modreanu, J. Sanho-Parramon, D. O'Connell, J. Justice, O. Durand, and B. Servet, Mater. Sci. Eng., B 118, 127 (2005).

${ }^{45}$ S.-G. Lim, S. Kriventsov, T. N. Jackson, J. H. Haeni, D. G. Schlom, and A. M. Balbasov, J. Appl. Phys. 91, 4500 (2002).

${ }^{46}$ P. W. Peacock and J. Robertson, J. Appl. Phys. 92, 4712 (2002).

${ }^{47}$ H. Takeuchi, D. Ha, and T.-J. King, J. Vac. Sci. Technol. A 22, 1337 (2004).

${ }^{48}$ H. Jin, S. K. Oh, H. J. Kang, and M.-H. Cho, Appl. Phys. Lett. 89, 122901 (2006).

${ }^{49}$ F. J. Himpsel, F. R. McFeely, A. Teleb-Ibrahimi, J. A. Yarmoff, and G. Hollinger, Phys. Rev. B 38, 6084 (1988).

${ }^{50}$ J. W. Keister, J. E. Rowe, J. J. Kolodziej, H. Niimi, T. E. Madey, and G. Lucovsky, J. Vac. Sci. Technol. B 17, 1831 (1999).

${ }^{51}$ M. Oshima, S. Toyoda, T. Okumura, J. Okabayashi, H. Kumigashira, K. Ono, M. Niwa, K. Usuda, and N. Hirashita, Appl. Phys. Lett. 83, 2172 (2003).

${ }^{52}$ O. Renault, N. T. Barrett, D. Samour, and S. Quiais-Marthon, Surf. Sci. 566-568, 526 (2004)

${ }^{53}$ K. Xiong, J. Robertson, M. C. Gibson, and S. J. Clark, Appl. Phys. Lett. 87, 183505 (2005).

${ }^{54}$ J. L. Gavartin, D. Munoz Ramo, A. L. Schluger, G. Bersuker, and B. H. Lee, Appl. Phys. Lett. 89, 082908 (2006).

${ }^{55}$ K. Shiraishi, K. Yamada, K. Torii, Y. Akasaka, K. Nakajima, M. Konno, T. Chikyo, H. Kitajima, and T. Arikado, Jpn. J. Appl. Phys., Part 2 43, L1413 (2004).

${ }^{56}$ K. Shiraishi, H. Takeuchi, Y. Akasaka, H. Watanabe, N. Umezawa, T. Chikyow, Y. Nara, T.-J. King Liu, and K. Yamada, Proceedings of the IEEE International Conference on Simulation of Semiconductor Processes and Devices, 2006 (unpublished), p. 306.

${ }^{57}$ M. Houssa, S. D. Gendt, J. L. Autran, and G. Groeseneken, Appl. Phys. Lett. 85, 2101 (2004).

${ }^{58}$ V. V. Afanas'ev and A. Stesmans, J. Appl. Phys. 95, 2518 (2004).

${ }^{59}$ N. Capron, P. Broqvist, and A. Pasquarello, Appl. Phys. Lett. 91, 192905 (2007).

${ }^{60}$ V. V. Afanas'ev and A. Stesmans, J. Appl. Phys. 102, 081301 (2007). 